\title{
JunB differs from c-Jun in its DNA- binding and dimerization domains, and represses c-Jun by formation of inactive heterodimers
}

\author{
Tiliang Deng and Michael Karin ${ }^{1}$
}

Department of Pharmacology, Center for Molecular Genetics, University of California, San Diego, School of Medicine, La Jolla, California 92093-0636 USA

JunB differs considerably from c-Jun in its ability to activate AP-1-responsive genes and induce oncogenic transformation. We demonstrate that the decreased ability of JunB to activate gene expression is the result of a small number of amino acid changes between its DNA-binding and dimerization motifs and the corresponding regions of $c$-Jun. These changes lead to a 10-fold decrease in the DNA-binding activity of JunB. JunB can be converted into a c-Jun-like activator by substituting four amino acids in its DNA-binding and dimerization motifs with the corresponding c-Jun sequences. JunB can also attenuate trans-activation by c-Jun, an activity mediated by its leucine zipper. This ability depends on two glycine residues that decrease the stability of the JunB leucine zipper, resulting in decreased homodimerization and increased heterodimerization. These results illustrate how small changes in primary structure, including chemically conservative changes, can result in functional divergence of two highly related transcriptional regulators.

[Key Words: JunB; c-Jun; DNA-binding/dimerization motifs; leucine zipper]

Received October 16, 1992; revised version accepted December 29, 1992.

Transcriptional control is commonly mediated by sequence-specific DNA-binding proteins (Pabo and Sauer 1984; Johnson and McKnight 1989). Many such proteins, both prokaryotic and eukaryotic, associate with their cognate DNA recognition motifs as either homodimers or heterodimers with structurally related proteins. Two large families of dimeric eukaryotic transcription factors were identified: the bZip (basic-zipper) and the bHLH (basic-helix-loop-helix) proteins (Landschulz et al. 1988a; Abel and Maniatis 1989; Johnson and McKnight 1989; Murre et al. 1989; Olson 1990). These proteins regulate a variety of physiological functions, including cell proliferation and differentiation, and their activity is modulated by various cytokines and growth factors $1 \mathrm{Ol}-$ son 1990; Karin 1991). The DNA-binding domains of both protein families consist of a basic region rich in positively charged amino acids, which interacts directly with the DNA, and an adjacent dimerization domain (Landschulz et al. 1988a; Murre et al. 1989). Structural models suggest that the basic mode of DNA recognition is conserved between both of these protein families (Vinson et al. 1989; O'Neil et al. 1990; Anthony-Cahill et al. 1992, Vinson and Garcia 1992). The bZip and bHLH proteins can interact with other family members through their dimerization motifs and thereby expand their regulatory potential.

\footnotetext{
${ }^{1}$ Corresponding author.
}

AP-1 was identified as a transcription factor required for optimal activity of the human metallothionein IIA promoter in vitro and in vivo (Haslinger and Karin 1985; Scholer et al. 1986; Karin et al. 1987; Lee et al. 1987a). It was later shown to mediate gene induction in response to various activators of protein kinase $\mathrm{C}$, such as $12-\mathrm{O}$ tetradecanoyl-phorbol-13-acetate (TPA) (Angel et al. 1987; Lee et al. 1987b). AP-1 is a bZip protein whose constituents belong to the Jun and Fos protein families (for review, see Angel and Karin 1991). Although the Jun proteins bind to the TPA response element (TRE) as homodimers or Jun-Jun heterodimers, the Fos proteins fail to bind this site on their own because they cannot form stable homodimers or Fos-Fos heterodimers. However, the Fos proteins can form heterodimers with the various Jun proteins, which are more stable than Jun-Jun dimers and, therefore, exhibit higher DNA-binding activity (Nakabeppu et al. 1988; Smeal et al. 1989).

The Jun proteins, c-Jun, JunB, and JunD, are very similar to each other, especially in their DNA-binding and transcriptional activation domains (Vogt and Bos 1990). Earlier in vitro studies suggested that the ability of the Jun proteins to interact with c-Fos and the TRE is quite similar (Nakabeppu et al. 1988). It was therefore assumed that all three proteins serve the same physiological function. However, the iun genes were found to be expressed in different sites both in adult mice and during embryogenesis (Hirai et al. 1989; Wilkinson et al. 1989; Mellström et al. 1991) and respond to different signal 
transduction pathways (Chiu et al. 1989). These findings raised the possibility that the three jun gene products may have different functions. However, it is also formally possible that the three Jun proteins have identical biochemical activities and that multiple genes exist only to increase the flexibility of their regulation in response to multiple environmental signals, which are too diverse and complex to modulate the expression of a single gene. The same questions regarding physiological function apply to other bZip and bHLH proteins that can interact with common sequence motifs. Each of these proteins appears to have highly similar relatives.

In the case of the Jun proteins, it was found that despite their similar primary structures (Vogt and Bos 1990) and DNA-binding specificities (Nakabeppu et al. 1988; Ryseck and Bravo 1991), they differ greatly in their abilities to activate transcription of AP-1-dependent promoters (Chiu et al. 1989; Yang-Yen et al. 1990) and induce transformation of either rodent (Schutte et al. 1989) or avian (Castellazzi et al. 1991) fibroblasts. The first indication of different functions was found for c-Jun and JunB; whereas c-Jun is an effective activator of the human collagenase promoter, as well as a synthetic promoter containing a single TRE, JunB is rather ineffective (Chiu et al. 1989). Furthermore, coexpression of JunB with c-Jun repressed trans-activation by the latter protein (Chiu et al. 1989|. In addition, coexpression of JunB decreased the transforming activity of c-Jun (Schutte et al. 1989).

The functional differences between c-Jun and JunB are quite intriguing in light of the great similarity between their DNA-binding, dimerization, and transcriptional activation domains (Vogt and Bos 1990). The similarity between the primary structures of the two proteins offers a unique opportunity for a reversed genetic analysis of the basis for these differences. By constructing and analyzing a large number of c-Jun/JunB chimeras, we found that the decreased activity of JunB is caused by a small number of amino acid substitutions between its leucine zipper and basic regions and the corresponding c-Jun sequence. Most important, two naturally occurring glycine residues within the leucine zipper of JunB decrease its homodimerization ability. By preferential formation of heterodimers with decreased DNA-binding activity, JunB is capable of repressing c-Jun activity. These studies illustrate how small changes in primary structure can result in a large variation in the activities of two closely related transcriptional regulators and are likely to be of relevance to other bZip and bHLH proteins that, despite a high degree of sequence similarity, exhibit different biological activities.

\section{Results}

Differential gene activation by $c$-Jun and Jun $B$ dictated by their DNA-binding domains

c-Jun and JunB have similar primary structures (Vogt and Bos 1990|. From sequence comparison, it is not obvious which region of the two proteins determines their different biological activities. To determine the structural basis for these differences, we performed domain swap ex- periments. Our initial efforts were concentrated on the amino-terminal regions of c-Jun and JunB, because earlier results suggested that this region may determine the different activities of the two proteins (Chiu et al. 1989). The domain swaps used in these earlier studies introduced short insertions or deletions into the resulting chimeric proteins that could potentially affect their function in a nonphysiological manner. To avoid this problem, a series of chimeric c-Jun/JunB and JunB/c-Jun proteins was constructed in which no deletion or insertion of any amino acid has occurred and, therefore, is likely to suffer only a minimal structural perturbation. These chimeras were constructed by swapping fragments of c-Jun and JunB cDNAs using either naturally occurring or artificially introduced restriction sites located within highly conserved regions. All of the chimeric cDNAs, as well as the two wild-type cDNAs, were inserted into a mammalian expression vector using the strong promoter within the long terminal repeat (LTR) of Rous sarcoma virus (RSV). The wild-type and chimeric expression vectors were cotransfected into F9 cells, a mouse embryonal carcinoma cell line that exhibits a very low level of endogenous AP-1 activity (Chiu et al. 1988), with two different AP-1 responsive reporter plasmids. As shown in Figure 1, expression of wild-type c-Jun led to efficient (55- to 60-fold) trans-activation of either -73 Col-CAT, which contains $73 \mathrm{bp}$ of the human collagenase promoter $(\mathrm{Col})$ fused to chloramphenicol acetyltransferase (CAT) structural sequence (Angel et al. 1987), or -79 jun-CAT, which contains $79 \mathrm{bp}$ of the human c-jun promoter fused to the CAT gene (Angel et al. 1988). Both of these promoter regions contain AP-1 sites. As reported earlier (Chiu et al. 1989), only a low level of trans-activation (three- to fivefold) of either reporter was achieved by cotransfection of the wild-type JunB expression vector. Transfection with increasing amounts of the JunB expression vector did not result in further activation of either -73 Col-LUC (Figure 2) or -79 jun-LUC (data not shown). These differences are not attributable to differences in the expression level of the two proteins (Chiu et al. 1989; see also Fig. 5 below). All of the c-Jun/JunB (CB) chimeras that contained c-Jun sequences from amino acid 1 to either 71 (CB1), 117 (CB2), 169 (СB3), or 246 (CB4) behaved like JunB, whereas the JunB/c-Jun $(\mathrm{BC})$ chimeras containing the corresponding Jun $\mathrm{B}$ amino-terminal sequences, $\mathrm{BCl}$, $\mathrm{BC} 2, \mathrm{BC} 3$, or BC4, behaved like c-Jun. Amino acid 246 is located immediately upstream of the DNA-binding domains of both c-Jun and JunB. In contrast to the results obtained with the previously constructed JunB/c-Jun chimera (Chiu et al. 1989), the present findings suggested that the different activities of c-Jun and JunB may be the result of differences in their DNA-binding domains. Since the previous results, based on a JunB/c-Jun chimera containing the 236 amino-terminal residues of JunB and the last 109 carboxy-terminal residues of c-Jun, are fully reproducible (data not shown), we conclude that the behavior of that particular chimera is most likely the result of a structural alteration that does not affect the chimeras used in the present study. 


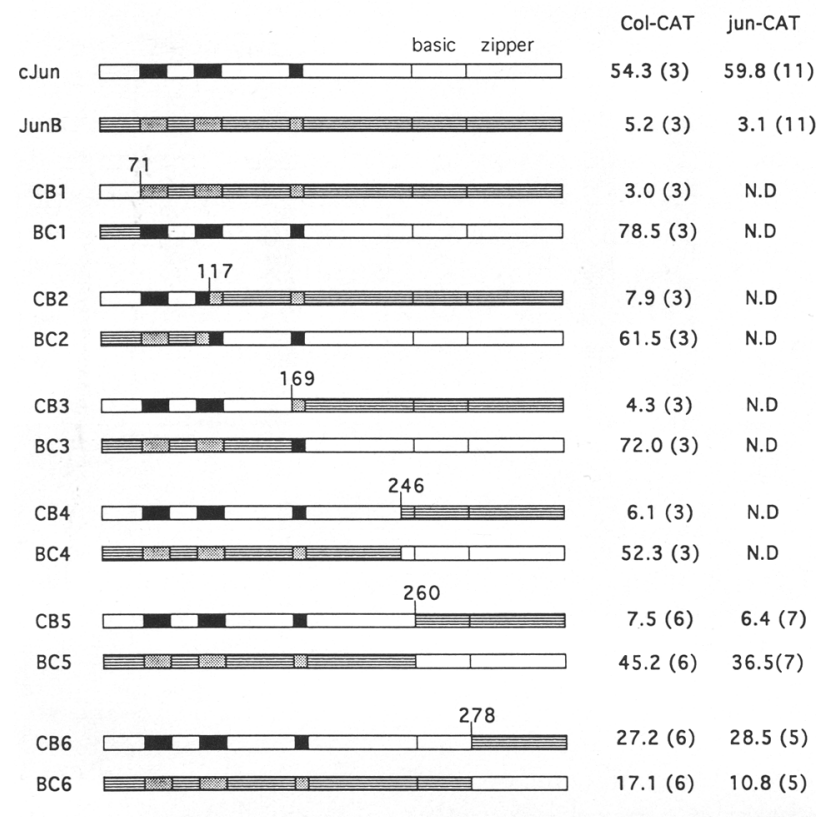

Figure 1. Transactivation by wild-type c-Jun, JunB, and the various c-Jun/JunB and JunB/c-Jun chimeras. A schematic representation of c-Jun, JunB, and the various chimeras is shown. The black boxes in c-Jun denote the three previously identified subregions of its activation domain (Angel et al. 1989), and the dotted boxes in JunB denote the corresponding sequences. Trans-activation by the various constructs is indicated as fold activation above basal level (obtained with empty expression vector). (N.D.) Not done. F9 cells were transfected with the indicated reporters $(2 \mu \mathrm{g} /$ plate $)$ and expression vectors $(1 \mu \mathrm{g} /$ plate), and CAT activity was determined 12-16 hr later. The results are the means of several experiments the number of which are indicated in parenthesis.

To further examine the role of the DNA-binding domain, we generated a restriction site at the sequence surrounding codons 260 of c-Jun and 273 of JunB, which correspond to the exact amino termini of their basic regions (Vogt and Bos 1990). As shown in Figure 1, the chimera containing amino acids $1-260$ of c-Jun and the JunB DNA-binding domain, CB5, was an inefficient activator of reporter gene expression as with JunB, whereas the reverse chimera, $\mathrm{BC} 5$, was a potent activator similar to c-Jun. A chimera containing the first 278 amino acids of c-Jun, CB6, whose basic region is derived from c-Jun, but whose leucine zipper is from JunB was twofold less efficient than c-Jun. Hence, the JunB leucine zipper may not be as effective as the corresponding region of c-Jun. This finding is further supported by the significant increase in activity of the reverse chimera, BC6, which contains mostly JunB sequences with the exception of the leucine zipper derived from c-Jun; in comparison with wild-type JunB, BC6 was 3.5 -fold more active. In summary, these results indicate that the major determinants of the differential activity of c-Jun and JunB are located in the basic region, which directly contacts DNA, and the leucine zipper, which mediates dimerization (Vinson et al. 1989).
To further investigate the relative contribution of the DNA-binding and activation domains to the differential activity of c-Jun and JunB, the following experiments were undertaken. First, the amino-terminal halves of c-Jun and JunB were separately fused to a heterologous DNA-binding domain derived from the yeast activator Gal4 (amino acids 1-147; Sadowski and Ptashne 1989) to generate chimeric Gal4-c-Jun (1-246) and Gal4-JunB (1258) proteins (Fig. 3A). Although the lengths of the c-Jun and JunB sequences fused to the Gal4-binding domain are slightly different, their carboxy-terminal end points are located in the same position relative to their DNAbinding domains (Vogt and Bos 1990). We found that the trans-activating potentials of both chimeras were very similar (Fig. 3B). Second, we fused the activation domain located in the 78 carboxy-terminal amino acids of the viral activator VP16 (Sadowski et al. 1988) to the carboxy-terminal halves of either c-Jun or CB4 (Fig. 3A). The resultant chimeric proteins contain the DNA-binding domain of either c-Jun or JunB, respectively. Consistent with the results shown in Figure 1, the VP16-c-Jun chimera was a much better activator of a -79 jun-luciferase (LUC) reporter than the VP16-CB4 chimera (Fig. $3 \mathrm{C})$. The difference in the activities of both chimeras is very similar to the difference between c-Jun and JunB. Collectively, these results confirm that the distinct behavior of c-Jun and JunB is mostly the result of structural and functional differences between their DNA-binding domains.

Four amino acid changes convert JunB into $a$ c-Jun-like activator

To identify which an:ino acid substitutions between the

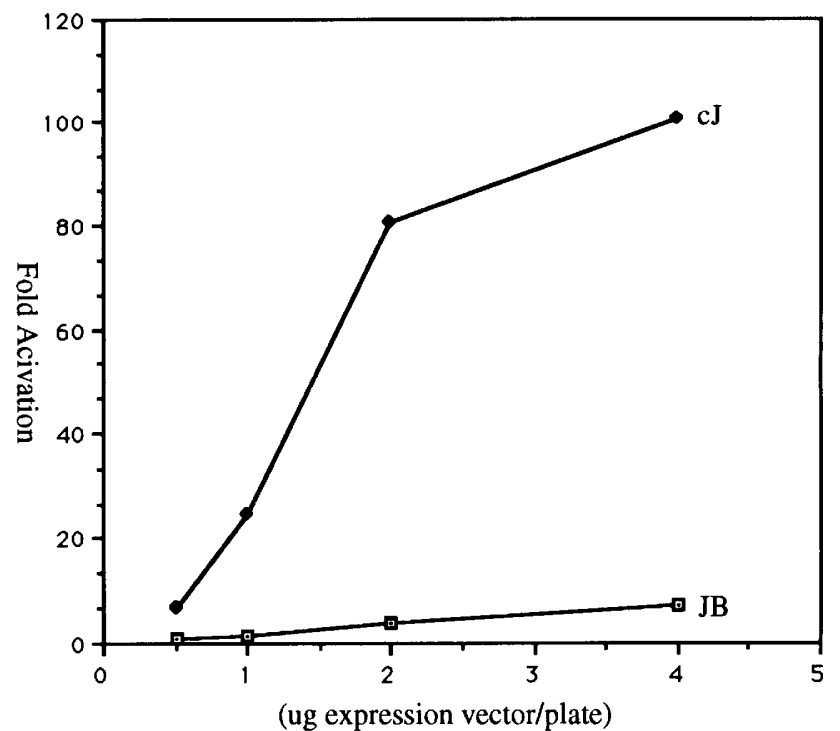

Figure 2. Dose-dependent activation by c-Jun and JunB. The indicated amounts of the c-Jun and JunB expression vectors were cotransfected into F9 cells with $2 \mu \mathrm{g}$ of a -73 Col-LUC reporter. Luciferase activity was determined $16 \mathrm{hr}$ later. The results shown are the averages of two experiments. 
A

\author{
Activators: \\ Gal4-cJun(1-246) \\ Gal4-JunB(1-258) \\ VP16-cJun
}

VP16-CB4
B

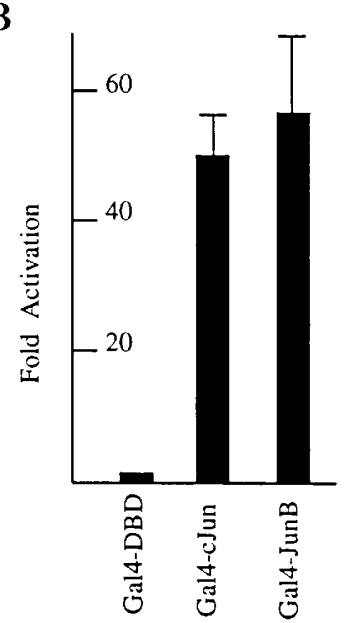

C

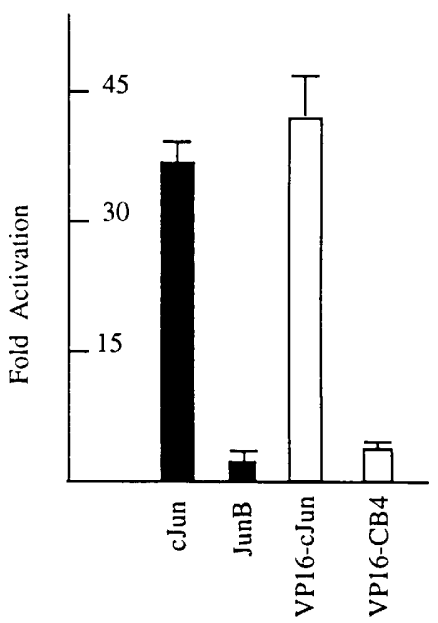

Figure 3. Differential activation by c-Jun and JunB is the result of differences between their DNA-binding domains. $(A)$ Schematic representation of the chimeric Gal4-c-Jun, Gal4-JunB, VP16-c-Jun, and VP16-CB4 activators used in these experiments. (B) F9 cells were transfected with a $1 \times$ Gal4-E 1 b-LUC reporter $(2 \mu \mathrm{g} / \mathrm{plate})$ and the indicated expression vectors $(0.5 \mu \mathrm{g} / \mathrm{plate})$ and luciferase activity was determined $12-16 \mathrm{hr}$ later. The results shown are the means of three experiments (each done in duplicate) and are presented as fold increases in luciferase activity over the baseline seen with the reporter and Gal4(1-147). (C) F9 cells were transfected with -79 iun-LUC $(2 \mu \mathrm{g} / \mathrm{plate})$, and the indicated expression vectors $(1 \mu \mathrm{g} / \mathrm{plate})$ and luciferase activity were determined $12-16 \mathrm{hr}$ later. The results are the means of four experiments (each done in duplicate) and are presented as fold increases in luciferase activity over the baseline seen with -79 iun-LUC alone.

DNA-binding domains, including both the basic regions and leucine zippers, of c-Jun and JunB are responsible for their differential activity, we replaced JunB-specific sequences with the corresponding c-Jun sequences. The alignment of the two DNA-binding domains is shown in Figure $4 \mathrm{~A}$, and the amino acid residues that differ between the two proteins are in boldface type. The activities of the various mutants measured by their ability to activate the -79 jun-CAT reporter are indicated in Figure 4B.

Among the 11-amino-acid differences between the two DNA-binding domains, all except two, E293G and N299G, are conservative changes. Interestingly, these sequence substitutions result in the presence of two glycines within the leucine zipper of JunB. Glycine and proline residues are helix destabilizers and are discriminated against within leucine zippers (Landschulz et al. 1988a). Therefore, these glycines are likely to decrease the stability of the $\alpha$-helix formed by the JunB leucine zipper. Replacement of the two glycines of JunB with the corresponding c-Jun sequences, resulted in a four- to fivefold increase in activity (Fig. 4B). Replacement of either glycine alone resulted in a smaller increase in JunB activity (data not shown). Two of the sequence differences, I264L and S267T, between the DNA-binding domains of c-Jun and JunB, reside in the basic region. Despite the conserved nature of these changes, replacement of the JunB sequences with the corresponding c-Jun sequences [JB(L264I/T267S)], resulted in a six- to sevenfold increase in activity. The effect of the single substitutions was smaller, with T267S being more effective than L264I. Combination of the two basic region substitutions with the two leucine zipper substitutions resulted in a 12-fold
A

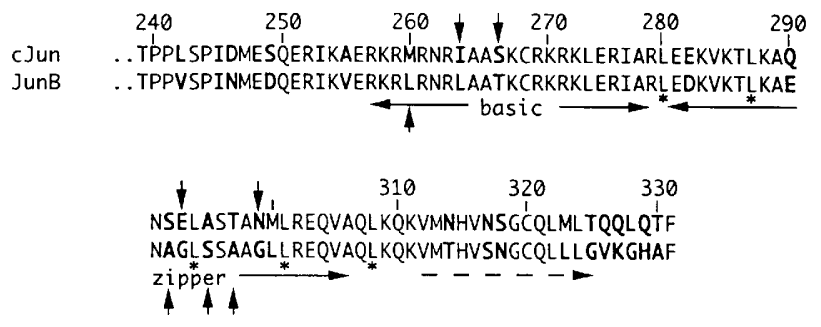

B

$\begin{array}{lc}\text { Proteins } & \text { Fold Activation } \\ \text { CJun WT } & 59.8(11) \\ \text { JunB WT } & 3.1(11) \\ \text { JB L264I } & 4.9(4) \\ \text { JB T267S } & 10.7(9) \\ \text { JB L264I } & 19.9(5) \\ \text { T267S } & \\ \text { JB G293E } & 14.3(4) \\ \text { G299N } & \\ \text { JB L264I } & 35.8(4) \\ \text { T267S } & \\ \text { G293E } & \\ \text { G299N } & \end{array}$

Figure 4. JunB can be converted to a c-Jun-like activator. $|A|$ Sequence comparison between the DNA-binding domains of c-Jun and JunB. The amino acid differences between the two proteins are highlighted in boldface type. The amino acid substitutions that had a large effect on the trans-activation ability of JunB are indicated by the top arrows. The amino acid substitutions that had no effect on the trans-activation ability of JunB are indicated by the bottom arrows. $(B)$ The various wild-type and mutant expression vectors were cotransfected with the -79 jun-CAT reporter as described in Fig. 1 and the fold activation is indicated. The number of experiments used to calculate these values are indicated in parenthesis. 
increase in JunB activity, reaching almost the same level of activity as c-Jun. To confirm the importance of the two glycine substitutions within the leucine zipper, we converted three other positions within the JunB leucine zipper to the corresponding c-Jun sequences (A292S, S295A, and A297T). Individually, none of these substitutions had any effect on JunB activity (data not shown).

A trivial explanation of the results shown in Figures 1 and 4 is that the increased activity is the result of stabilization of JunB resulting in elevated expression. This explanation, however, is ruled out by the results shown in Figure 5. Immunoprecipitation analysis of two representative JunB mutants, expressed in transfected F9 cells indicated that the amino acid substitutions did not increase the expression level of JunB(L264I/T267S/ G293E/G299N) compared with that of wild-type JunB. However, the presence of c-Jun-derived carboxy-terminal sequences resulted in a twofold increase in the level of BC4 expression in comparison with the level of JunB expression. This twofold increase in expression is unlikely to account for the 10-fold increase in activity displayed by BC4. (Please note that because of the use of different antibodies, the expression levels of the various JunB proteins cannot be precisely compared with the level of c-Jun expression.)

\section{JunB DNA-binding domain is responsible for repression of c-Iun activity}

In addition to its much lower trans-activation potential, JunB is capable of repressing trans-activation and transformation by c-Jun (Chiu et al. 1989; Schutte et al. 1989). Three simple mechanisms may explain the repression of

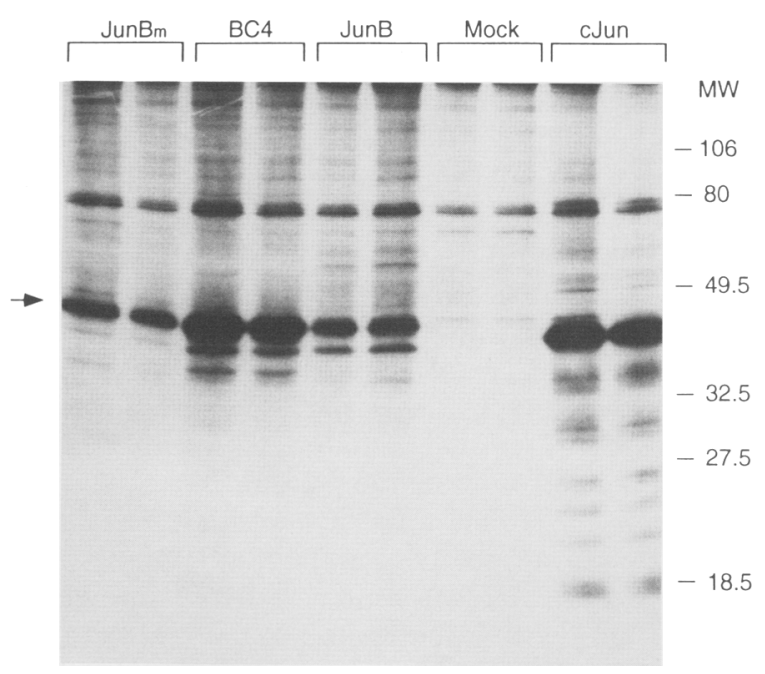

Figure 5. Immunoprecipitation analysis of Jun protein expression. Expression vectors encoding wild-type c-Jun, wild-type JunB, a JunB/c-Jun chimera (BC4) and a mutant JunB(L264I/ T267S/G293E/G299N) (JunBm), and a control plasmid (pUC18; mock) were transfected into F9 cells and analyzed as described in Materials and methods with c-Jun- and JunB-specific antibodies. The results of two separate experiments are shown. The Jun- specific band is indicated by the arrow:
c-Jun activity by JunB. First, JunB may compete with c-Jun for binding to the TRE, resulting in replacement of an effective activator (c-Jun) by a noneffective one (JunB). Second, c-Jun and JunB may preferentially form heterodimers, which may or may not bind to the TRE. If the heterodimer does not bind to the TRE, obviously it cannot stimulate transcription. If the heterodimer binds to the TRE, it may do so with reduced affinity or be defective in transcriptional activation. Third, JunB may compete with c-Jun for an interaction with a cofactor required for activation, and for some reason the complex formed between JunB and the cofactor is much less active than the cofactor-c-Jun complex. A clue to the possible mechanism of repression can be obtained by determining which part of JunB is responsible for repression of c-Jun activity. We examined the ability of the various chimeras to repress c-Jun activity and found that although CB1, CB2, CB3, CB4, and CB5 were efficient repressors, CB6 was not (data not shown). These results suggested that the JunB DNA-binding domain is required for efficient repression of c-Jun activity. These results were confirmed by titration experiments using either wild-type JunB or a truncated JunB expression vector, specifying a protein consisting of its carboxy-terminal half and lacking its activation domain (Jun $\Delta A$ ), which indicated that the JunB DNA-binding domain is sufficient for efficient repression of c-Jun activity (Fig. 6A). These results suggest that JunB does not repress c-Jun by sequestering a cofactor that interacts with its aminoterminal activation domain. Further argument against a squelching mechanism is provided by the titration data shown in Figure 2. No self-squelching could be observed even in rather high inputs of either c-Jun or JunB. To determine how the DNA-binding domain of JunB acts to repress c-Jun, we constructed a chimeric activator containing the activation domain and basic region of c-Jun and the leucine zipper of CCAAT/enhancer-binding protein- $\alpha(\mathrm{C} / \mathrm{EBP} \alpha)$. This chimeric activator should dimerize, bind to the TRE with the same specificity as c-Jun, and activate transcription of AP-1-dependent promoters. However, because C/EBP $\alpha$ does not dimerize with any of the Jun or Fos proteins (Cao et al. 1991), the cJ/CEBP chimera should not heterodimerize with JunB, yet it should be capable of competing with JunB for binding to the TRE. As shown in Figure 6B, the cJ/CEBP chimera can stimulate transcription of the -79 jun-LUC reporter as efficiently as c-Jun. However, unlike c-Jun, it is only weakly repressed by JunB, even when an eightfold molar excess of a JunB expression vector was used. The weak repression of cJ/CEBP activity by JunB is most likely the result of squelching, because unlike c-Jun, cJ/ CEBP was not repressed by the truncated JunB protein, $J u n B \Delta A$. Collectively, these results suggest that repression of c-Jun activity by JunB requires heterodimerization of the two proteins.

\section{JunB is an inefficient DNA-binding protein and represses c-Jun DNA binding}

To investigate the biochemical basis for the decreased activity of JunB and its ability to repress c-Jun, we com- 
A

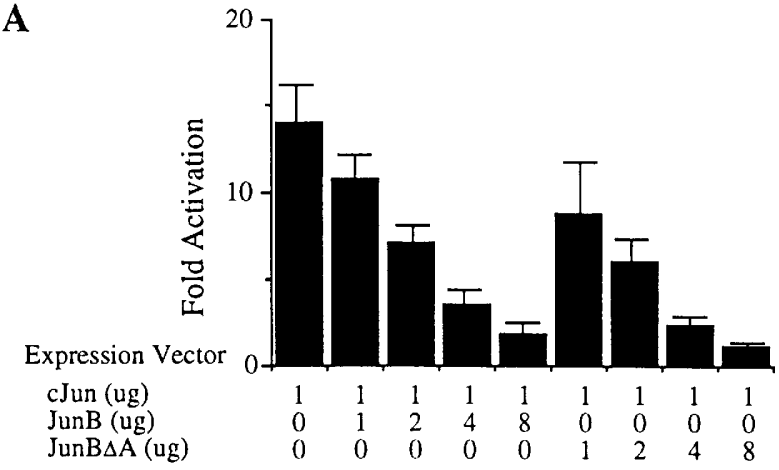

B

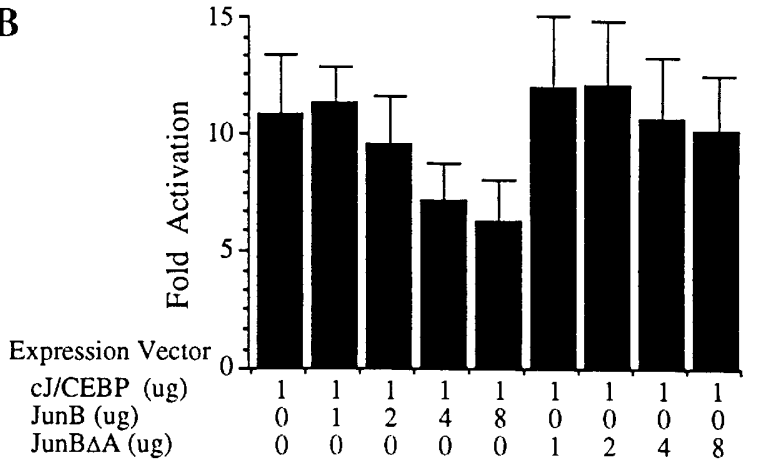

Figure 6. Repression of c-Jun and cJ/CEBP activity by JunB and truncated JunB containing only its DNA-binding domain (JunB $\Delta \mathrm{A}$ ). (A) The -79 jun-LUC reporter $(2 \mu \mathrm{g} /$ plate) was cotransfected with $1 \mu \mathrm{g}$ of the c-Jun expression vector in the absence or presence of the indicated amounts of the JunB (wild type) and JunB $\Delta A$ expression vectors. The results are shown as fold activation of reporter gene expression and represent the means of four separate experiments. $(B)$ The -79 jun-LUC reporter $(2 \mu \mathrm{g} / \mathrm{plate})$ was cotransfected with one of the cJ-CEBP expression vectors in the absence or presence of the indicated amounts of the JunB and JunB $\Delta A$ expression vectors. The results shown are the means of four separate experiments.

pared the DNA-binding activity of recombinant c-Jun and JunB proteins produced in Escherichia coli. Previous experiments indicated that recombinant c-Jun is fully functional in DNA-binding (Deng and Karin 1992) and transcriptional activation (C. Lai, unpubl.). Despite the ease of expression and purification of intact c-Jun (Deng and Karin 1992), we found it difficult to express fulllength JunB in $E$. coli. Therefore, we chose to express a c-Jun/JunB chimera, CB4, because it exhibited the same activation and repression properties as JunB. Unlike JunB, CB4 can be expressed as a full-length protein in $E$. coli (Fig. 7A). The proteins were purified and their binding to a radioactively labeled c-jun TRE probe was examined by mobility-shift assays (Fried and Crothers 1981). The results of experiments in which the c-jun TRE was kept at a constant concentration ( $3.3 \mathrm{nM})$ and incubated with increasing concentrations of either c-Jun or CB4 are shown in Figure 7B. Half-maximal binding of the TRE probe was achieved at $4.3 \mathrm{nM}$ of c-Jun and $45 \mathrm{nM}$ of CB4. These results indicate that JunB is much less efficient than c-Jun in DNA-binding and can explain its reduced trans-activation potential. Furthermore, reconstitution of the four amino acid substitutions that convert the JunB into a c-Jun-like activator (L264I, T267S, G293E, G299N) into CB4, resulted in a protein that bound the TRE as efficiently as c-Jun. Half-maximal binding by CB4(L264I/T267S/G293E/G299N) was observed at $4.5 \mathrm{~nm}$ protein. These results are in general agreement with those of Ryseck and Bravo (1991), who found that c-Jun is a more effective DNA-binding protein than JunB. However, as their experiments were based on cell-free translated proteins, no direct quantitation of DNA-binding was performed.

The results shown in Figure 6 suggested that JunB represses c-Jun by heterodimer formation. To investigate this possibility further, we incubated equimolar amounts of c-Jun with a truncated JunB protein (t-JunB), or truncated c-Jun (tc-Jun) with JunB. The various dimeric complexes formed between these ${ }^{35} \mathrm{~S}$-labeled proteins were trapped by using the irreversible crosslinker disuccinimidyl suberate (DSS). Cross-linking of the $c$-Jun and $t$-JunB mixture resulted in formation of c-Jun : c-Jun homodimers and c-Jun : $t$-JunB heterodimers, but hardly any t-JunB : $t$-JunB homodimers (Fig. $8 \mathrm{~A})$. Similar results were obtained in the reverse experiment; cross-linking of an equimolar JunB + tc-Jun mixture resulted in formation of JunB : tc-Jun heterodimers, which were most abundant, followed by tc-Jun : tc-Jun homodimers, whereas hardly any JunB : JunB homodimer could be detected. Even when tested on its own, c-Jun was found to homodimerize more efficiently than JunB. After quantitation and normalization for their different methionine contents, the c-Jun: JunB heterodimers were found to form approximately twofold more readily than the c-Jun : c-Jun homodimers. The preferential heterodimer formation is most likely driven by the monomeric state of JunB, as suggested by the very low amount of JunB : JunB homodimers.

We then examined DNA-binding by an equimolar mixture of CB4 and tc-Jun. As shown in Figure 8B, despite preferential heterodimer formation confirmed by cross-linking experiments (data not shown), the amount of the jun TRE probe bound by tc-Jun : tc-Jun homodimers was much higher than the amount of probe bound by CB4 : tc-Jun heterodimers. Very little probe bound to the $\mathrm{CB} 4$ homodimers. Other experiments indicate that tc-Jun and $t$-JunB have similar DNA-binding activity to their respective full-length proteins (Fig. 8C). Collectively, these results indicate that although incubation of JunB with c-Jun results in preferential heterodimer formation, the resultant heterodimers do not bind to the TRE as efficiently as c-Jun homodimers. This indicates that JunB can repress c-Jun by forming heterodimers with decreased DNA-binding activity.

\section{Discussion}

Previous studies have shown differences in expression 
A

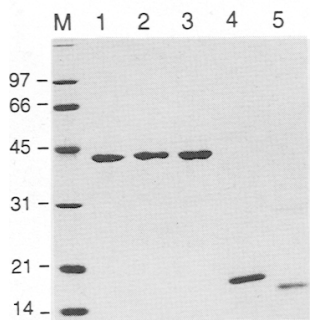

B
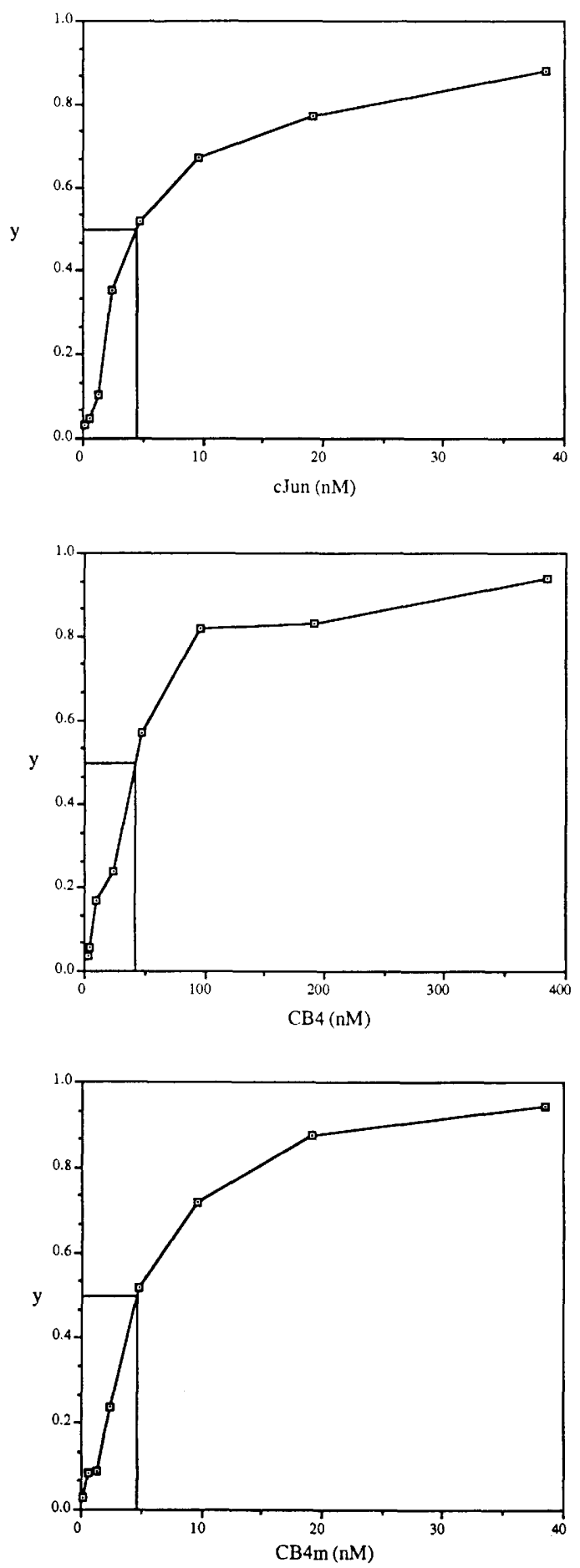

patterns and response to extracellular stimuli between the c-iun and junB genes (Bartel et al. 1989; Hirai et al. 1989; Pertovaara et al. 1989; Ryder et al. 1989; Wilkinson et al. 1989; Mellström et al. 1991). Although originally the products of these genes were reported to exhibit similar DNA-binding specificity and heterodimer formation with c-Fos (Nakabeppu et al. 1988); they were later shown to differ considerably in their trans-activation and transformation abilities, with c-Jun being far more active than JunB (Chiu et al. 1989; Schütte et al. 1989). Sequence comparison indicates that most of the amino acid differences between the c-Jun and JunB are located in their amino-terminal halves (Vogt and Bos 1990), which in the case of c-Jun contain the major transcriptional activation domain (Angel et al. 1989). On the other hand, the carboxy-terminal DNA-binding domains are highly conserved. It was anticipated, therefore, that the functional differences between c-Jun and JunB are the result of the differential activity of their amino-terminal activation domains, an assumption that was supported by the reduced activity of a JunB/c-Jun chimera (Chiu et al. 1989). Therefore, the results of this study, which indicate that JunB differs from c-Jun because of a small number of structural changes within its DNAbinding domain, are rather surprising. These conclusions are not only based on the analysis of a series of c-Jun/ JunB chimeras but also on the analysis of VP16-c-Jun and VP16-JunB fusions. Those proteins that contain the DNA-binding domain of c-Jun were found to be 10 -fold more active, in vivo, than the proteins that contain the DNA-binding domain of JunB, regardless of the origin of their activation domain. The differences in DNA-binding activity were found to be attributable to four amino acid changes between c-Jun and JunB. By substituting these four amino acids in JunB with the corresponding c-Jun residues, it was possible to convert JunB to an efficient transcriptional activator in vivo and an effective DNA-binding protein in vitro. These results are of general importance because they illustrate clearly how small differences in primary sequences, including chemically conservative changes, can generate transcriptional activators with divergent biochemical and biological activities.

Figure 7. DNA-binding by recombinant c-Jun and JunB proteins. (A) SDS-PAGE analysis of the bacterially produced and purified proteins used in these experiments, stained with Coomassie blue. (Lane 1), c-Jun; (lane 2), CB4; (lane 3), CB4(L264I/T267S/G293E/G299N); (lane 4), t-Jun; (lane 5), $\mathrm{t}$-JunB. (M) molecular mass markers. (B) DNA-binding curves. A fixed concentration ( $3.3 \mathrm{nM}$ ) of end-labeled jun-TRE probe was incubated with increasing concentrations of c-Jun, CB4, and CB4 (L264I/T267S/G292E/G299N), as indicated (in nM). The DNA-protein complexes were resolved on a nondenaturing polyacrylamide gel, and the amount of bound and free probe was directly quantitated using the Ambis radioanalytic imaging system. Average values of fractional occupancy $(y)$ from three different experiments were plotted as a function of protein concentration. The concentration of each protein required to shift $50 \%$ of the probe is indicated. 

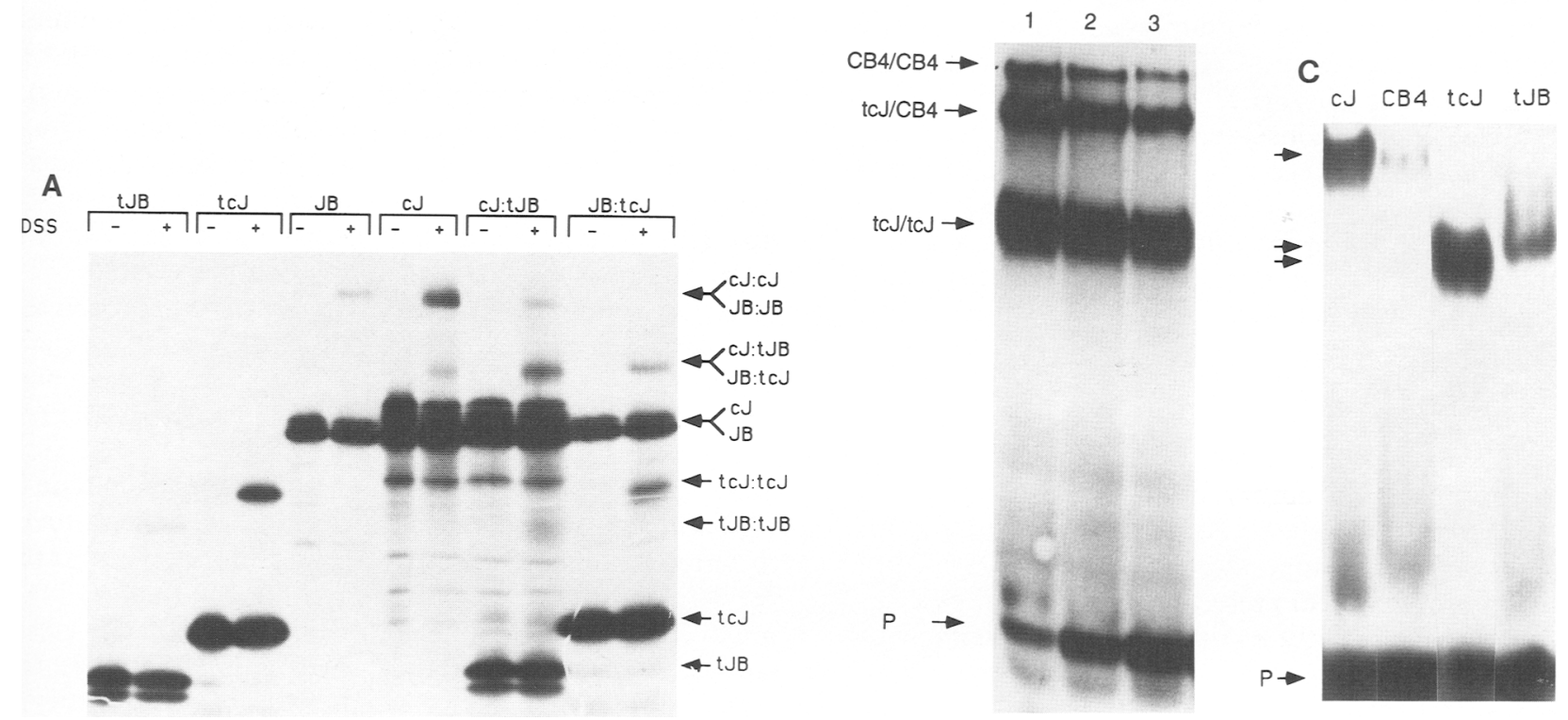

Figure 8. Preferential formation of c-Jun : JunB heterodimers with reduced DNA-binding activity. $|A|$ Analysis of heterodimer formation. Equimolar amounts of cell-free translated, ${ }^{35}$ S-labeled, c-Jun (cJ), JunB (JB), truncated c-Jun (tcJ) and truncated JunB (tJB), proteins, either alone or in combination with each other, were preincubated under the same conditions used for measuring DNAbinding activity in the absence of an exogenous DNA probe. The mixtures were then incubated with dimethylsulfoxide (DMSO) (-) or DSS in DMSO $(+1$, as described in Materials and methods, and analyzed by SDS-PAGE and autoradiography. The migratrion positions of the monomers and the different homodimeric and heterodimeric complexes are indicated. $(B)$ Equimolar concentrations of CB4 (45 ng/reaction) and t-Jun ( $15 \mathrm{ng} /$ reaction) were mixed with a ${ }^{32} \mathrm{P}$-labeled jun-TRE probe (1 ng/reaction) in the absence (lane 1) or presence of 5 -fold (lane 2) or 10-fold (lane 3) excess of cold jun-TRE. The migration positions of the different protein-DNA complexes and the free probe $(\mathrm{P})$ are indicated. $(C)$ Forty nanograms of c-Jun (cJ), CB4, truncated c-Jun (tcJ), and truncated JunB (t JBB were incubated with ${ }^{32}$ P-labeled jun-TRE probe, as indicated above, and analyzed by electrophoresis on a native polyacrylamide gel. The migration positions of the different protein DNA complexes and the free probe $(\mathrm{P})$ are indicated by the arrows.

Two of the major changes between c-Jun and JunB are the substitutions of E293 and N299 of c-Jun by glycines in JunB. The effect of these changes on the activity of JunB is relatively easy to understand. Sequence comparison indicates that glycines and prolines, both of which are known to be helix destabilizers, are infrequently found within leucine zippers (Landschulz et al. 1988a), which are known to form stable $\alpha$-helices (O'Shea et al. $1989,1991)$. Therefore, the two glycines are expected to decrease the stability of JunB leucine zipper. Indeed, the replacement of both of these glycines with the corresponding c-Jun sequences increases the activity of JunB by four- to fivefold and is essentially as effective as substitution of the entire JunB leucine zipper with the corresponding region of c-Jun. Furthermore, the cross-linking experiments indicate that JunB homodimerizes much less efficiently than c-Jun. The decreased homodimerization activity of JunB also explains the preferential formation of $\mathrm{c}$-Jun : JunB heterodimers. Although the reduced homodimerization activity of JunB is not as severe as that of $c$-Fos, the interaction between JunB and c-Jun is analogous to the interaction between c-Fos and c-Jun. O'Shea et al. (1992) have recently shown that the major driving force behind the preferential formation of c-Jun : c-Fos heterodimers is the monomeric state of c-Fos. By mass action, when mixed with c-Jun homodimers, the c-Fos monomers drive the mixture into heterodimer formation (O'Shea et al. 1989, 1992). This is apparently the case for JunB and c-Jun: the c-Jun : JunB heterodimer forms twofold more readily than the c-Jun : c-Jun homodimer. Preferential heterodimer formation explains why JunB is such an efficient repressor of c-Jun activity. Once the specificity of the c-Jun leucine zipper is changed so that it can no longer interact with JunB, c-Jun becomes relatively resistant to inhibition by elevated expression of JunB.

The effect of the substitution of 1264 and S267 of c-Jun by leucine and threonine, respectively, in JunB is more difficult to understand. These conservative changes combined are responsible for a six- to sevenfold difference in the activities of the two proteins. Although the substitution of isoleucine with leucine is expected to increase $\alpha$-helical stability, the substitution of serine with threonine should decrease helix stability (Lyu et al. 1990; O'Neil and DeGrado 1990). Therefore, it is difficult to predict the combined effect of these substitutions on the stability of the $\alpha$-helix formed by the basic region, once bound to DNA $10^{\prime}$ Neil et al. 1990; Talanian et al. 1990; Weiss et al. 1990). One possible explanation is that these changes, which affect residues that are not directly 
involved in DNA-binding (K. Struhl, pers. comm.), lead to a slight distortion in the positions occupied by the adjacent alanine residues, both of which have a key role in sequence recognition ( $\mathrm{K}$. Struhl, pers. comm.). Because the residues that are involved in sequence recognition are absolutely conserved between c-Jun and JunB, it is unlikely that the two proteins recognize different sequences. In any case, complete understanding of how the I264L and T267S substitutions affect DNA-binding by JunB will have to wait until a high-resolution structure of a Jun-TRE complex is available. Together, the leucine zipper and the basic region changes lead to a 10 -fold decrease in the DNA-binding activity of JunB determined in vitro and an almost 20 -fold decrease in its trans-activation potential determined in vivo. Furthermore, the decreased ability of JunB to interact with the TRE results in decreased DNA-binding by the c-Jun : JunB heterodimer. This decreased DNA-binding activity, combined with preferential heterodimer formation, can account for the repression of c-Jun activity by JunB.

The results obtained with c-Jun and JunB clearly demonstrate how small changes in primary structure can result in functional divergence within a family of highly related transcriptional regulators. In this case, sequence divergence within the dimerization/DNA-binding domain led to the appearance of a protein that is not only relatively inefficient in homodimerization and DNAbinding but is also capable of forming stable heterodimers with a related family member that have decreased DNA-binding activity. These changes, as we demonstrated, are important for JunB becoming a negative regulator of $\mathrm{c}$-Jun and provide strong support to the notion that JunB is functionally inequivalent to c-Jun and that its major function is to be an attenuator of c-Jun. It was recently found that inactivation of the c-jun gene by homologous recombination results in early embryonic lethality, indicating that $j u n B$ and $j u n D$ cannot substitute for c-jun (E. Wagner, B. Spiegelman, pers. comm.). However, it is also possible that the lack of complementation of c-jun by the two other jun genes is attributable to differences in their expression pattern.

There are many other examples of structurally related transcriptional regulators with highly divergent functions. For example, the IRF2 protein that is closely related to the activator of type I interferon genes, IRF1, inhibits interferon gene activation by IRFl (Harada et al. 1989). Although IRF2 has a DNA-binding domain that is more effective than IRF1, it has a weak activation domain. Therefore, IRF2 appears to inhibit IRF1 activity by competition for a common DNA-binding site. Other repressors, such as Id and I-POU, which are members of the bHLH and POU families, respectively, lack certain residues involved in DNA-binding and, therefore, have no intrinsic DNA activity. Yet they have functional dimerization domains and can inhibit DNA-binding by other family members through formation of inactive heterodimers (Benezra et al. 1990; Treacy et al. 1991).

The mechanism of c-Jun repression by JunB is more similar to the mechanism of repression used by Id or
I-POU than the mechanism of repression used by IRF2. Unlike IRF2, which is more effective in DNA-binding than IRF1, JunB binds DNA less efficiently than c-Jun. Therefore, JunB cannot compete with c-Jun for the AP-1 site. Rather, as discussed above, JunB interacts with c-Jun to form heterodimers with reduced DNA-binding activity. However, JunB differs from Id and I-POU because it is not totally defective in DNA-binding. Although it binds DNA rather weakly on its own, it can form heterodimers with c-Fos whose DNA-binding activity is considerably higher than the activity of JunB homodimers (Nakabeppu et al. 1988; Ryseck and Bravo 1991). Although the JunB : c-Fos heterodimer does not activate target gene expression as efficiently as the c-Jun : c-Fos heterodimer, or even c-Jun: c-Jun homodimers, it is considerably more active than the JunB : JunB homodimer (Yang-Yen et al. 1990). Therefore, in the absence of c-Fos, JunB would act as a repressor of AP-1 activity by attenuating trans-activaton by c-Jun, whereas in the presence of c-Fos, the preferential formation of JunB : c-Fos heterodimers should contribute to AP-1 activity.

As mentioned earlier, most of the amino acid differences between c-Jun and JunB proteins are located in their amino-terminal activation domains, yet the present results indicate that the two domains have similar activity. However, we have shown previously that c-Jun and JunB differ in their ability to respond to $\mathrm{Ha}$ Ras; unlike c-Jun, trans-activation by JunB is not potentiated by Ha-Ras (Binetruy et al. 1991). Recent analysis indicates that the ability to respond to Ha-Ras is determined by sequences located in the amino-terminal activation domain of c-Jun (Smeal et al. 1991) that are missing in JunB (T. Deng unpubl.). Both JunB(L264I/T267S/ G293E/G299N) and the BC4 chimera, which have very similar basal activity to c-Jun, are unresponsive to $\mathrm{Ha}$ Ras (T. Deng, unpubl.) Because transformation by c-Jun requires cooperation with Ha-Ras (Schütte et al 1989), these differences may contribute, in addition to the decreased DNA-binding activity, to the negligible transforming activity of JunB. Preliminary results indicate that despite its increased DNA-binding and trans-activation activities, the $\mathrm{BC} 4$ chimera has very low transforming activity, not considerably different from that of wildtype JunB (M. Birrer and T. Deng unpubl.). Experiments are in progress to determine the exact structural changes responsible for this decrease in transforming activity and their mechanism of action.

\section{Materials and methods}

\section{Cell culture and transfections}

F9 cells were grown in a 1:1 mixture of Dulbecco's modified Eagle medium (DMEM) and F12 supplemented with $10 \%$ fetal bovine serum and $10^{-4} \mathrm{M} \beta$-mercaptoethanol. Cells were split 3-4 hr before transfection by the calcium phosphate coprecipitation procedure as described previously (Angel et al. 1988, 1989). The total amount of transfected DNA was generally kept at $10 \mu \mathrm{g} / 100-\mathrm{mm}$ plate. 


\section{Reporters}

The $-73 /+63$ Col-CAT, $-79 /+170$ jun-CAT, and $1 \times$ Gal4 Elb-CAT reporters were described previously (Angel et al. 1988; Chiu et al. 1989; Lillie and Green 1989). p19LUC (van Zonneveld et al. 1988) was modified by deleting a 497-bp DraII fragment (from 20 to 5693) and replacing its AlwNI-AatII fragment with the corresponding fragment of pUC18. The new plasmid, named p20LUC, is a higher copy number and smaller plasmid than p19LUC. The HindIII-BamHI fragments of $-73 /+63$ Col-CAT, $-79 /+170$ jun-CAT and $1 \times$ Gal4-Elb-CAT, containing their promoter regions, were inserted into the polylinker region of p20LUC between the HindIII and the downstream BamHI sites to generate the $-79 /+170$ jun-LUC and $1 \times$ Gal4-Elb-LUC reporters, respectively.

\section{Site-directed mutagenesis}

To generate c-Jun templates, the $550 \mathrm{bp}$ XhoII-PstI fragment of RSV-c-Jun was subcloned into pBluescript KS|+|II, and the 550 bp PstI-NotI fragment of RSV-c-Jun was subcloned into pBluescript SK $(+)$. To generate the JunB templates, the 660-bp HindIII-SacI fragment of RSV-JunB was subcloned into M13mpl9, and the 600-bp SacI-XhoI fragment of RSV-JunB was subcloned into pBluescript $\mathrm{KS}|+| \mathrm{II}$. Mutagenesis was performed on singlestranded templates using the appropriate oligodeoxynucleotides (whose description is available upon request) and the Amersham site-directed mutagenesis kit as recommended by the manufacturer. The mutations were initially identified by creation or loss of restriction sites or by sequencing. Final confirmation was by direct sequencing. The appropriate mutants were subcloned back into their respective expression vectors.

\section{Chimeric activators}

RSV-c-Jun and RSV-JunB were described previously (Angel et al. 1988; Chiu et al. 1989). RSV-JunB $\Delta A$ was generated by deleting the 501-bp StyI-PvuII fragment of RSV-JunB. The junction sequence was determined to make sure that the deletion was in-frame. The chimeric c-Jun/JunB and JunB/c-Jun activators were generated by using restriction sites that exist in both expression vectors in identical positions or by creating new restriction sites in at least one of the two expression vectors by site-directed mutagenesis. To generate $\mathrm{CBl}$ and $\mathrm{BCl}$, an NheI site was introduced into the amino-terminal region of $c-J u n$, and the resulting mutant was named RSV-c-Jun(C1). The HindIIINheI fragment, containing the $5^{\prime}$-untranslated region and the amino-terminal region of RSV-JunB was replaced with the corresponding region of $\mathrm{RSV}-\mathrm{c}-\mathrm{Jun}(\mathrm{Cl})$ to generate $\mathrm{CB} 1$, whereas $\mathrm{BCl}$ was generated by the converse replacement. To generate $\mathrm{CB} 2$ and $\mathrm{BC} 2$, an Eco47III site was created in the amino-terminal coding regions of both c-Jun and JunB, and the resulting mutants were named RSV-c-Jun(C2) and RSV-JunB(B2), respectively. CB2 was obtained by replacing the HindIII-Eco47III of RSV-JunB(B2) with the corresponding region of RSV-c-Jun(C2), whereas $\mathrm{BC} 2$ was made by the converse replacement. $\mathrm{CB} 3$ and $\mathrm{BC} 3$ were generated by using a conserved $A c c \mathrm{I}$ site present in both c-Jun and JunB, whereas CB4 and BC4 were generated using a conserved BstXI site, using the approach described above. To generate CB5 and BC5, an SphI site was introduced into the basic region of $c$-Jun and JunB, and resulting mutants were named RSV-c-Jun(C5) and RSV-JunB(B5). In the process of creating RSV-JunB(B5), a conserved amino acid change (Leu to Met) occurred. This is the only case among all the mutants used to generate the chimeras where an amino acid change was introduced. However, this alteration had no effect on the activity of RSV-JunB(B5) in comparison with wild-type RSV-JunB (data not shown). To generate $\mathrm{CB} 6$ and $\mathrm{BC} 6$, a $B s s \mathrm{HII}$ site corresponding to a naturally occurring BssHII site in JunB, was created at the junction of the basic region and leucine zipper region of c-Jun. The resulting mutant was named RSV-c-Jun(C11). CB5 and BC5 and $\mathrm{CB} 6$ and BC6 were obtained by the same approach used for the other chimeras.

To generate RSV-VP16-c-Jun, a HindIII-RsaI fragment from Gal4-VP16 was inserted between the HindIII and AccI sites of RSV-c-Jun. The RsaI-AccI junction was sequenced to make sure that the reading frame was maintained. The resulting construct was named Gal4-VP16-c-Jun. The NotI-XbaI fragment of pECE-flag (Ellis et al. 1986) was cloned into pBluescript $\mathrm{SK}|+|$ to generate a single-stranded template. The template was mutagenized to generate a $B s p H I$ site just upstream of the $B g l I I$ site in the flag-coding sequence. The mutated plasmid was named pECE-flag2. A 396-bp XhoII-PstI fragment of Gal4 VP16-c-Jun, which codes for 3 amino acids in the linker region upstream of the VP16-coding region (amino acids 413-488), the VP16 activation domain and a part of c-Jun, was inserted into pECE-flag2 between its Bg/II and PstI sites. A 401-bp BspHI$P$ stI fragment was then used to replace the amino-terminal coding region of RSV-c-Jun (C6). RSV-VP16-CB4 was generated by replacing a HindIII-BstXI fragment from RSV-CB4 for the corresponding fragment of RSV-VP16-c-Jun.

To generate Gal4-c-Jun or Gal4-BC4, a 680-bp EcoRI-Sall fragment of pSG5-T3R $\alpha$ (Sap et al. 1990) was inserted into pSG424 between its EcoRI and Sall sites to generate Gal4T3R $\alpha-$ RS. To generate Gal4-c-Jun, the BspHI-TaqI fragment of RSV-c-Jun(C6), encoding amino acids 1-246 of c-Jun, was used to replace the NcoI-SalI fragment of Gal4-T3R $\alpha-$ RS. The Gal4 JunB chimera was generated by the same approach.

To generate c-Jun-CEBP, a BamHI-SacI fragment of murine sarcoma virus (MSV)-C/EBP (Landschulz et al. 1988b) was cloned into pBluescript $\mathrm{SK}|+|$ to generate a single-stranded template that was mutagenized to create a BssHII site [C/E] mutation) in the junction that separates the basic region and the leucine zipper region of C/EBP. The BamHI-HindIII fragment of MSV-C/EBP was cloned into pBluescript SK $1+1$. The XbaI$X$ hoI fragment of the resulting plasmid was then cloned into RSV-JunB to replace the coding region of JunB with that of $\mathrm{C} / \mathrm{EBP}$. The new construct is designated RSV-C/EBP. The C/E1 mutation was cloned back into the RSV-C/EBP to generate RSV-C/EBP(C/E1). The 890-bp BssHII (partial)-XhoI of RSV$\mathrm{C} / \mathrm{EBP}(\mathrm{C} / \mathrm{E} 1)$ and 1070-bp HindIII-BssHII (partial) of RSV-cJun $(\mathrm{C} 11)$ were ligated with a $3.9-\mathrm{kb}$ HindIII-XhoI fragment of RSV-JunB to generate RSV-cJ/CEBP(BssHII).

\section{Expression and purification of recombinant proteins}

The expression and purification of $\mathrm{c}$-Jun and $\mathrm{t}$-Jun were described previously (Deng and Karin 1992). pET-8c/c-Jun (Deng and Karin 1992) was modified by deleting the $3^{\prime} 1070$-bp untranslated region from $\mathrm{NarI}$ to $\mathrm{BamHI}$. The t-Jun-coding region has been transferred to this new plasmid. The modification improves the expression level of $\mathrm{t}$-Jun and, therefore, its purification. To adapt the JunB cDNA to the pET-8c vector /Studier et al. 1991), two nucleotides preceding its initiator ATG codon were mutated to create an AflIII site. The AfIIII (partial)-BamHI fragment from RSV-JunB(B16) was inserted into pET-8c between the NcoI and BamHI sites. The plasmid has also been modified by deleting the 1120-bp 3'-untranslated region from NarI to BamHI. To express t-JunB, a PstI site (B9) was introduced into JunB in the region immediately upstream of its basic region. The PstI-BamHI of RSV-JunB(B9) was subcloned into pET-8c/t-Jun. The NarI-BamHI 3'-untranslated region was removed as described above. All of the mutant JunB proteins were 
produced by replacing the SacI-Bsu36I fragments of pET-8c/ JunB with those of the various RSV-JunB mutants.

\section{Mobility-shift assay}

For mobility-shift assays (Fried and Crothers 1981), the different Jun proteins were incubated with $1 \mathrm{ng}$ of ${ }^{32} \mathrm{P}$-labeled TRE probe in the presence of $10 \mathrm{ng}$ of poly[d(I-C)], in $20 \mu \mathrm{l}$ of binding buffer containing $12 \mathrm{~mm}$ HEPES-KOH(pH 8.0), $50 \mathrm{~mm} \mathrm{KCl}, 6 \mathrm{~mm}$ $\mathrm{MgCl}_{2}, 1 \mathrm{~mm}$ EDTA, 10\% glycerol, $5 \mathrm{~mm} \mathrm{DTT}$, and $0.5 \mathrm{mg} / \mathrm{ml}$ of BSA. After a 20 -min incubation at room temperature, reaction mixtures were loaded onto $5 \%$ native polyacrylamide gels (acrylamide-bisacrylamide $40: 1$ ). Electrophoresis was performed in $0.4 \times \mathrm{TBE}$ at room temperature. Gels were dried and exposed to X-ray films at room temperature. The mobility-shift experiments were quantified by counting the dried gels with the Ambis radioanalytic imaging system. The sequence of the junTRE is

\section{5'-AGCTTGGTGACATCATCCG ACCACTGT AGT AGGCCT AG-5'}

\section{Immunoprecipitation}

F9 cells were transfected with $10 \mu \mathrm{g}$ of the various expression vectors and labeled for $12 \mathrm{hr}$ after transfection for $3 \mathrm{hr}$ with $\left[{ }^{35} \mathrm{~S}\right]$ methionine $(100 \mu \mathrm{Ci} / \mathrm{ml})$. The levels of Jun protein synthesis was determined by immunoprecipitation using c-Jun or JunBspecific antibodies as described (Binetruy et al. 1991; Boyle et al. 1991). The immunoprecipitates were analyzed by SDS-PAGE, and the dried gel was quantitated by the Ambis radioanalytic imaging system.

\section{In vitro translation and chemical cross-linking}

To generate in vitro-translated proteins, $X b a \mathrm{I}-\mathrm{BamHI}$ fragments of cDNAs from bacterial expression vectors $\mathrm{pET}-8 \mathrm{c} / \mathrm{c}$ Jun, $\mathrm{pET}-8 \mathrm{c} / \mathrm{t}$-Jun, $\mathrm{pET}-8 \mathrm{c} / J u n B$ or $\mathrm{pET}-8 \mathrm{c} / \mathrm{t}$-JunB were cloned into pSP64-poly(A) for use in Promega's TNT-coupled reticulocyte lysate system. The proteins were produced in the presence of $\left[{ }^{35} \mathrm{~S}\right]$ methionine according to the manufacturer's instructions. Two microliters of each sample was analyzed on $12 \%$ denaturing SDS-PAGE gel. The gel was fixed and treated with Amplify (Amersham) according to the supplier's instructions. The dried gel was quantitated by the Ambis radioanalytic imaging system. Because the translational efficiency of each construct is different and each protein has a different number of methionine residues (c-Jun $=11$, JunB $=4, \mathrm{t}$-Jun $=7$, and $\mathrm{t}$-JunB $=3$ ), the volumes of the different translation reactions were adjusted to give equal concentrations of each protein. Equimolar mixtures of the different cell-free translated Jun proteins were prepared in $40 \mu \mathrm{l}$ of mobility-shift buffer, incubated at $45^{\circ} \mathrm{C}$ for $10 \mathrm{~min}, 37^{\circ} \mathrm{C}$ for $30 \mathrm{~min}$, and cross-linked by adding $1 \mu \mathrm{l}, 40 \mathrm{~mm}$ DSS for $20 \mathrm{~min}$ at room temperature. The reactions were quenched by adding $5 \mu \mathrm{l}$ of $1 \mathrm{M}$ lysine and analyzed and quantitated as described (Deng and Karin 1992).

\section{Acknowledgments}

We thank Dr. Joseph DiDonato for TNT coupled reticulocyte lysate systems, provided by Dr. Greg Beckler of Promega, Dr. Michael Birrer for oligonucleotides and transformation assays, and Ms. K. Sexton for secretarial assistance. This work was supported by grants from the National Institutes of Health (ES04151, CA50528) and the Cancer Research Coordination Committee of the University of California. T.D. was supported in part by a fellowship from the Tobacco-Related Disease Research Program of California.
The publication costs of this article were defrayed in part by payment of page charges. This article must therefore be hereby marked "advertisement" in accordance with 18 USC section 1734 solely to indicate this fact.

\section{References}

Abel, T. and T. Maniatis. 1989. Gene regulation: Action of leucine zippers. Nature 341: 24-25.

Angel, P. and M. Karin. 1991. The role of Jun, Fos and the AP-1 complex in cell-proliferation and transformation. Biochim. Biophys. Acta 1072: 129-157.

Angel, P., M. Imagawa, R. Chiu, B. Stein, R.J. Imbra, H.J. Rahmsdorf, C. Jonat, P. Herrlich, and M. Karin. 1987. Phorbol ester-inducible genes contain a common cis element recognized by a TPA-modulated trans-acting factor. Cell 49: 729-739.

Angel, P., K. Hattori, T. Smeal, and M. Karin. 1988. The Jun proto-oncogene is positively autoregulated by its product, Jun/AP1. Cell 55: 875-885.

Angel, P., T. Smeal, J. Meek, and M. Karin. 1989. Jun and v-Jun contain multiple regions that participate in transcriptional activation in an interdependent manner. New Biol. 1: 35-43.

Anthony-Cahill, S.J., P.A. Benfield, R. Fairman, Z.R. Wasserman, S.L. Brenner, W.F. Stafford III, C. Altenbach, W.L. Hubbell, and W.F. DeGrado. 1992. Molecular characterization of helix-loop-helix peptides. Science 255: 979-983.

Bartel, D., M. Sheng, L. Lau, and M. Greenberg. 1989. Growth factors and membrane depolarization activate distinct programs of early response gene expression: Dissociation of fos and jun induction. Genes \& Dev 3: 304-313.

Benezva, R., R.L. Davis, D. Lockshon, D.L. Turner, and H. Weintraub. 1990. The protein Id is a negative regulator of helix-loop-helix DNA-binding proteins. Cell 61: 49-59.

Binetruy, B., T. Smeal, and M. Karin. 1991. Ha-Ras augments c-Jun activity and stimulates phosphorylation of its activation domain. Nature 351: 122-127.

Boyle, W.J., T. Smeal, L.H.K. Defize, P. Angel, J.R. Woodgett, M. Karin, and T. Hunter. 1991. Activation of protein kinase C decreases phosphorylation of c-Jun at sites that negatively regulate its DNA-binding activity. Cell 64: 573-584.

Castellazzi, M., G. Spyrou, N. LaVista, J.-P. Dangy, F. Piu, M. Yaniv, and G. Brun. 1991. Overexpression of $c$-jun, junB, or junD affects cell growth differently. Proc. Natl. Acad. Sci. 88: 8890-8894.

Cao, Z., R.M. Umek, and S.L. McKnight. 1991. Regulated expression of three C/EBP isoforms during adipose conversion of 3T3-L1 cells. Genes \& Dev. 5: 1538-1552.

Chiu, R., P. Angel, and M. Karin. 1989. JunB differs in its biological properties from, and is a negative regulator of, c-Jun. Cell 59: 979-986.

Deng, T. and M. Karin. 1992. Construction and expression of a monomeric c-Jun protein that binds and activates transcription of AP-1-responsive genes. Proc. Natl. Acad. Sci. 89: 8572-8576.

Ellis, L., E. Clauser, D.O. Morgan, M. Edery, R.A. Roth, and W.J. Rutter. 1986. Replacement of insulin receptor tyrosine residues 1162 and 1163 compromises insulin-stimulated kinase activity and uptake of 2-deoxyglucose. Cell 45: 721-732.

Fried, M. and D. Crothers. 1981. Equilibrium and kinetics of lac repressor-operator interactions by polyacrylamide gel electrophoresis. Nucleic Acids Res. 9: 6505-6525.

Harada, H., T. Fujita, M. Miyamoto, Y. Kimura, M. Maruyama, A. Furia, T. Miyata, and T. Taniguchi. 1989. Structurally similar but functionally distinct factors, IRF-1 and IRTF-2, 
bind to the same regulatory elements of IFN and IFN-inducible genes. Cell 58: 729-739.

Haslinger, A. and M. Karin. 1985. Upstream promoter element of the human metallothionein-II $\lambda$ gene can act like an enhancer element. Proc. Natl. Acad. Sci. 82: 8572-8576.

Hirai, S.I., R.P. Ryseck, F. Mechta, R. Bravo, and M. Yaniv. 1989. Characterization of junD: A new member of the jun protooncogene family. EMBO I. 8: 1433-1438.

Johnson, P.F. and S.L. McKnight. 1989. Eukaryotic transcriptional regulatory proteins. Annu. Rev. Biochem. 58: 799839.

Karin, M. 1991. The AP-1 complex and its role in transcriptional control by protein kinase C. In Molecular aspects of cellular regulation (ed. P. Cohen and G. Foulkes). Vol. 6, pp. 143-161. Elsevier/North Holland Biomedical Press, Elsevier Publishers B.V. (Biomedical Division), Amsterdam, The Netherlands.

Karin, M., A. Haslinger, A. Heguy, T. Dietlin, and T. Cooke. 1987. The metal responsive elements act as positive modulators of human metallothionein-II $\lambda$ enhancer activity. Mol. Cell. Biol. 7: 606-613.

Landschulz, W.H., P.F. Johnson, and S.L. McKnight. 1988a. The leucine zipper: A hypothetical structure common to a new class of DNA-binding proteins. Science 240: 1759-1764.

Landschulz, W.H., P.F. Johnson, E.Y. Adashi, B.J. Graves, and S.L. McKnight. 1988b. Isolation of a recombinant copy of the gene encoding C/EBP. Genes 4 Dev 2: 786-800.

Lee, W., A. Haslinger, M. Karin, and R. Tjian. 1987a. Activation of transcription by two factors that bind promoter and enhancer sequences of the human metallothionein gene and SV40. Nature 325: 368-372.

Lee, W., P.J. Mitchell, and R. Tjian. 1987b. Purified transcription factor AP-1 interacts with TPA-inducible enhancer elements. Cell 49: 741-752.

Lillie, J.W. and M.R. Green. 1989. Transcription activation by the adenovirus E1A protein. Nature 338: 39-44.

Lyu, P.C., M.I. Liff, L.A. Marky, and N.R. Kallenbach. 1990. Side chain contributions to the stability of alpha-helical structure in peptides. Science 250: 669-673.

Mellström, B., M. Achaval, D. Montero, J.R. Navanjo, and P. Sassone-Corsi. 1991. Differential expression of the jun family memberes in rat brain. Oncogene 6: 1959-1964.

Murre, C., P.S. McCraw, H. Vassein, M. Caudy, L.Y. Jan, Y.N. Jan, C.V. Cabrera, J.N. Buskin, S.T. Hauschka, A.B. Lassar, H. Weintraub, and D. Baltimore. 1989. Interactions between heterologous helix-loop-helix proteins generate complexes that bind specifically to a common DNA sequence. Cell 58: 537-544.

Nakabeppu, Y., K. Ryder, and D. Nathans. 1988. DNA-binding activities of three murine jun proteins: Stimulation by fos. Cell 55: 907-915.

Olson, E. 1990. MyoD family: A paradigm for development? Genes \& Dev. 4: 1454-1461.

O'Neil, K.T. and W.F. DeGrado. 1990. A thermodynamic scale for the helix-forming tendencies of the commonly occurring amino acids. Science 250: 646-651.

O'Neil, K.T., R.H. Hoess, and W.F. DeGrado. 1990. Design of DNA-binding peptides based on the leucine zipper motif. Science 249: 774-778.

O'Shea, E.K., R. Rutkowski, and P.S. Kim. 1989. Evidence that the leucine zipper is a coiled coil. Science 243: 538-542.

O'Shea, E.K., J.D. Klemm, P.S. Kim, and T. Alber. 1991. X-ray structure of the GCN4 leucine zipper, a two-stranded, parallel coiled coil. Science 254: 539-544.

O'Shea, E.K., R. Rutkowski, and P.S. Kim. 1992. Mechanism of specificity in the Fos-Jun oncoprotein heterodimer. Cell 68:
699-708.

Pabo, C.O. and P.T. Sauer. 1984. Protein-DNA recognition. Ann. Rev. Biochem. 53: 293-321.

Pertovaara, L., L. Sistonen, T. Bos, P. Vogt, J. Keski-Oja, and K. Alitalo. 1989. Enhanced jun gene expression is an early genomic response to transformation growth factor $\beta$ stimulation. Mol.Cell.Biol. 9: 1255-1262.

Ryder, K., A. Lanahan, E. Perez-Albuerne, and D. Nathans. 1989. JunD: A third member of the Jun gene family. Proc. Natl. Acad. Sci. 86: 1500-1503.

Ryseck, R.F. and R. Bravo. 1991. c-JUN, JUN B, and JUN D differ in their binding affinities to AP-1 and CRE consensus sequences: Effect of FOS proteins. Oncogene 6: 533-542.

Sadowski, I and M. Ptashne. 1989. A vector for expressing Gal4(1-147) fusions in mammalian cells. Nucleic Acids Res. 17: 7539.

Sadowski, I., J. Ma, S. Triezeuberg, and M. Ptashne. 1988. Gal4 is an unusually potent transcriptional activator. Nature 335: 563-564.

Sap, J., L. de Magistris, H. Stunnenberg, and B. Vennstrom. 1990. A major thyroid hormone response element in the third intron of the rat growth hormone gene. EMBO I. 9: 887-896.

Scholer, H., A. Haslinger, A. Heguy, H. Holtgreve, and M. Karin. 1986. In vivo competition between a metallothionein regulatory element and the SV40 enhancer. Science 232: 76-80.

Schütte, J., J. Viallet, M. Nau, S. Segal, J. Fedorko, and J. Minna. 1989 jun- $B$ inhibits and $c$-fos stimulates the transforming and trans-activating activities of $c$-iun. Cell 59: 987-997.

Smeal, T., P. Angel, J. Meek, and M. Karin. 1989. Different requirements for formation of Jun : Jun and Jun : Fos complexes. Genes \& Dev 3: 2091-2100.

Smeal, T., B. Binetruy, D. Mercola, M. Birrer, and M. Karin. 1991. Oncogenic and transcriptional cooperation with HaRas requires phosphorylation of c-Jun on serines 63 and 73 . Nature 354: 494-496.

Studier. F.W., A.H. Rosenberg, J.J. Dunn, and J.W. Dubendorff. 1991. Use of T7 RNA polymerase to direct expression of cloned genes. Methods Enzymol. 185: 60-89.

Talanian, R.V., C.J. McKnight, and P.S. Kim. 1990. Sequencespecific DNA-binding by a short peptide dimer. Science 249: 769-773.

Treacy, M.N., X. He, and M. Rosenfeld. 1991. I-POU: A POUdomain protein that inhibits neuron-specific gene activation. Nature 350: 577--584.

van Zonneveld, A-J., S.A.Curriden, and D.J. Loskutoff. 1988. Type 1 plasminogen activator inhibitor gene: Functional analysis and glucocorticoid regulation of its promoter. Proc. Natl. Acad. Sci. 85: 5525-5529.

Vinson, C.R. and K.C. Garcia. 1992. Molecular model for DNA recognition by the family of basic-helix-loop-zipper proteins. New Biol. 4: 396-403.

Vinson, C.R., P.B. Sigler, and S.L. McKnight. 1989. Scissors-grip model for DNA recognition by a family of leucine zipper proteins. Science 246: 911-916.

Vogt, P.K. and T. J. Bos. 1990. jun: Oncogene and transcription factor. Adv. Cancer Res. 55: 1-35.

Weiss, M.A., T.E. Ellenberger, C.R. Wobbe, J.P. Lee, S.C. Harrison, and K. Struhl. 1990. Folding transition in the DNAbinding domain of GCN4 on specific binding to DNA. $\mathrm{Na}$ ture 347: 575-578.

Wilkinson, D.G., S. Bhatt, R-P. Ryseck, and R. Bravo. 1989. Tissue-specific expression of c-jun and jun B during organogenesis in the mouse. Development 106: 465-471.

Yang-Yen, H-F., R. Chiu, and M. Karin. 1990. Elevation of AP1 activity during F9 cell differentiation is due to increased c-jun transcription. New Biol. 2: 351-361. 


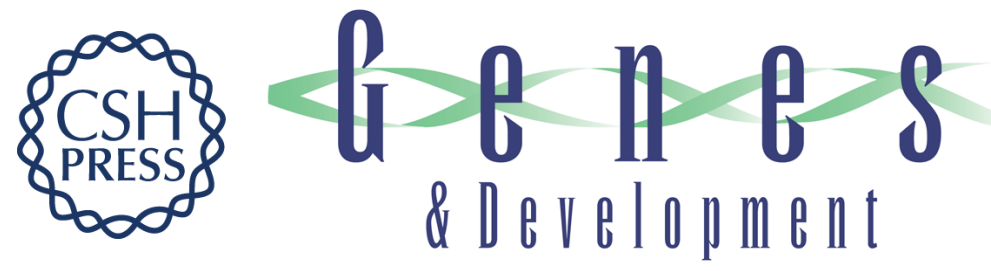

\section{JunB differs from c-Jun in its DNA-binding and dimerization domains, and represses c-Jun by formation of inactive heterodimers.}

T Deng and M Karin

Genes Dev. 1993, 7:

Access the most recent version at doi:10.1101/gad.7.3.479

References This article cites 56 articles, 23 of which can be accessed free at:

http://genesdev.cshlp.org/content/7/3/479.full.html\#ref-list-1

License

Email Alerting

Service

Receive free email alerts when new articles cite this article - sign up in the box at the top right corner of the article or click here.

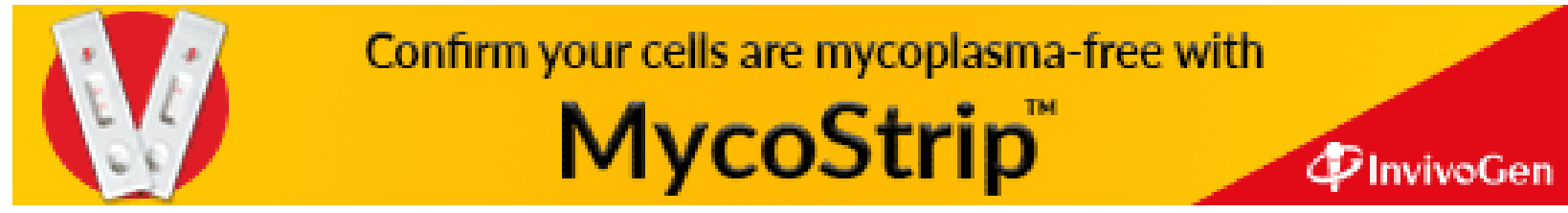

